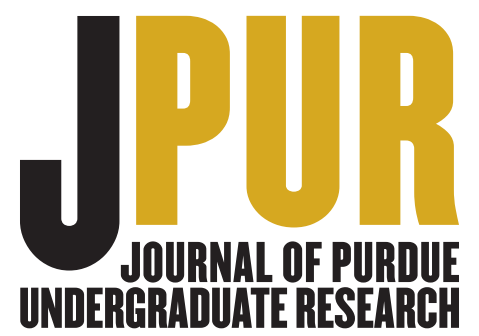

\title{
SCIENCE
}

\section{Uncovering the True Diversity of Chanterelles in Indiana}

\section{Student researcher: Jairus Chittenden, Sophomore}

Mushrooms in the genus Cantharellus, commonly known as chanterelles, are some of the most popular edible fungi in Indiana. Their vase-shaped orange and yellow fruiting bodies are a natural delicacy, and the global trade in chanterelles exceeds $\$ 1$ billion per year. A significant portion of chanterelles' true diversity remains undiscovered, and most collectors still use European species names for North American species. Chanterelles are challenging to classify based solely on morphological traits, as these can vary widely between members of the same species.

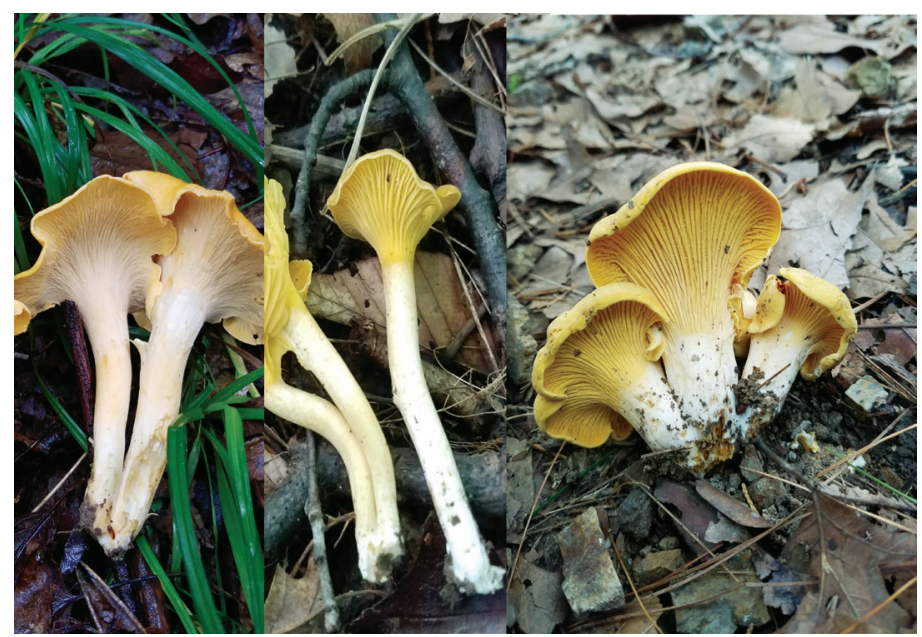

Left to center: Cantharellus flavolateritius and C. appalachiensis, two of seven species newly recorded in Indiana. Right: One of four potential new Indiana Cantharellus species.
Our goal is to use DNA sequence analyses to reassess the diversity of chanterelles in Indiana, using 130 specimens that have been collected as part of the North American Mycoflora Project. We extracted DNA from each specimen and used a polymerase chain reaction (PCR) to amplify the elongation factor 1-a (EF1a) protein-coding region. After editing, we were able to obtain 80 high quality sequences and match most of them to reference data. We constructed a phylogenetic tree using MUSCLE (a multiple sequence alignment algorithm) to confirm each identification.

Ten previously described chanterelle species are represented in our data so far, and seven of these were new records for Indiana. Eight specimens did not match previously described species with sufficient sequence identity and may represent as many as four undescribed species. In order to confirm these findings and expand our picture of chanterelle diversity even further, we will make more collections in 2019 and use another locus, the nuclear large subunit ribosomal DNA $(28 \mathrm{~S})$, to increase the resolution of our phylogenetic tree.

Research advisor M. Catherine Aime writes: "Of the 1.5 to 5.1 million conservatively estimated species of Fungi believed to be extant, less than 10\% have been described. Jairus's research is the first to examine species richness and distribution within the Midwest for members of the genus Cantharellus. Jairus's results show that the Midwest harbors much more fungal diversity than previously recognized, even in well-studied fungi such as the edible chanterelles." 